\title{
Epidemiology and Drug Resistance of Non Tuberculous Mycobacteria in India: a Mini Review
}

\author{
Tapti Sengupta*, Parijat Das and Tirthankar Saha \\ Department of Microbiology, West Bengal State University, India
}

Submission: March 17, 2016; Published: May 10, 2017

*Corresponding author: Tapti Sengupta, Department of Microbiology, West Bengal State University, India,Email: tapti.sg@gmail.com

\section{Introduction}

Nontuberculous mycobacteria are habitually environmental mycobacteria which are ubiquitous in nature residing in various environmental niche having pathogenic, non pathogenic and zoonotic characters [1,2]. The term nontuberculous mycobacteria (NTM) and mycobacteria other than tuberculosis (MOTT) originated from the concept that they comprise of Mycobacterium spp. which are not members of Mycobacterium tuberculosis complex. Earlier they were named as 'atypical mycobacterium' from the conviction that they are unusual and mostly non-pathogenic in comparison to Mycobacterium tuberculosis and Mycobacterium laprae strains. Recent investigations have recognized NTMs as potential causative agents for diseases in animals as well as human beings, especially in immuno compromised people [3]. Besides, NTM infections have been documented having zoonotic implications with its spread from fishes, birds and animals to human hosts, causing epidemics in nature. Environmental NTMs like $M$. fortuitum, M. gordonae, and M. chelonae has been screened from infected fishes and aquatic systems of Northern, Eastern and South Eastern Parts of India and most of them have been identified as human pathogens [4-6]. Apart from the eminent trouble of drug resistant tuberculosis (MTB) infections, NTM infections are now showing an elevated trend in India [7]. The clinical signs and symptoms of both NTM and MTB are related to granulomatous inflammations, which are quite similar and thus often go unrecognized and cannot be discriminated based on the common identification protocols of ATS (American Thoracic Society). Thus, NTM is easily misdiagnosed as M. tuberculosis, and multidrug-resistant (MDR), XDR (Extreme drug resistant) $\mathrm{TB}$, though the infection might be of multiple etiology. Several reports have come up in Indian context where NTMs have been reported in lung infections in patients thought to have tuberculosis $[7,8]$. Infections involving various pathogenic NTMs like M. marinum, M. fortuitum, $M$.chelonae have been reported in India $[9,10]$ similar to the reports worldwide. This shows the emerging trend of the Mycobacteria which were earlier neglected. With the rising incidences of antibiotic resistance in pathogens from environment and nosocomial habitats, these under documented microorganism needs limelight, as India is still fighting to overcome the Tuberculosis scenario. Resistance to multiple drug therapy was thought to be limited among Mycobacterium tuberculosis complex where MDR-TB is considered to be resistant to Isoniazid and (WHO, 2010). In recent years, nontuberculous mycobacterial infections and diseases have significantly increased with treatment strategies involving administration of the single or mixture of standard anti-tubercular drugs such as Rifampin, Isoniazid, Ethambutol, Streptomycin, Moxifloxacin, Telithromycin, Quinupristin and Dalfopristin [11]. Most of the clinical studies showed the NTMs to be remarkably resistant or only partially susceptible to these anti-tubercular drugs. The multi drug resistance of non tuberculous mycobacteria has now become a globally challenging health issue. NTMs encompass extensive diversity in phenotypic features depending on environmental distribution, growth rate, survival to environmental stress, colony morphology and coloration, biochemical characteristics and pathogenecity to eukaryotic hosts [12]. Among the different etiological habitats like soil, water, air, aquatic animals and human hosts, water plays the most important role as a natural vector or medium for mycobacterial disease transmission. Recently NTMs have increasingly been recognized as important opportunistic pathogens. Environmental mycobacterium residing in soil and water have been isolated from Northern and Eastern parts of India and most of these species have been reported as potential human pathogens $[6,7,9,10]$. In a study, mycobacteria were isolated from tap water, industrial polluted water, sewage, domestic garbage and drainage water and also from drinking water [13,14]. M. marinum, M. ulcerans and M. vaccae have been reported to cause skin infections [15]. M. fortuitum, M. gordonae, and $M$. chelonae were also described as potential fish pathogens causing ulceration in skin, parenchimal tissue and internal organs of ornamental and food fishes $[4,9,16,17]$.

Case reports involving NTM infections have been regularly documented. 
Biostatistics and Biometrics Open Access Journal

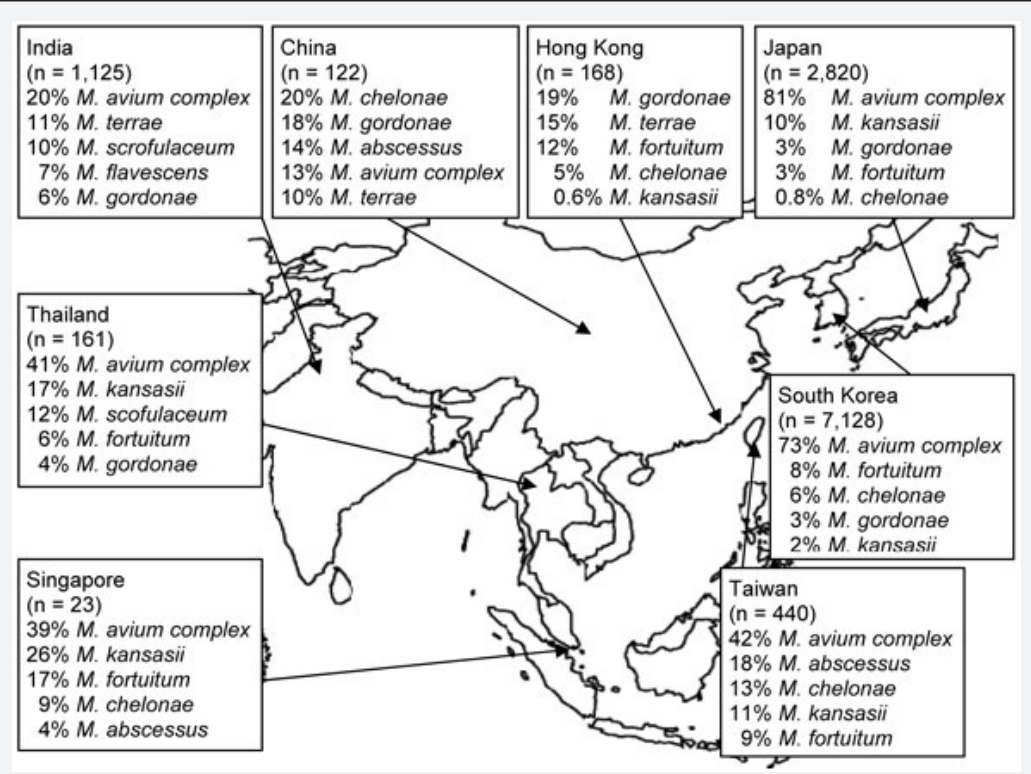

Figure 1: Five most prevalent nontuberculous mycobacteria species found in respiratory specimens, regardless of clinical relevance, Asia, 1971-2007 [17].

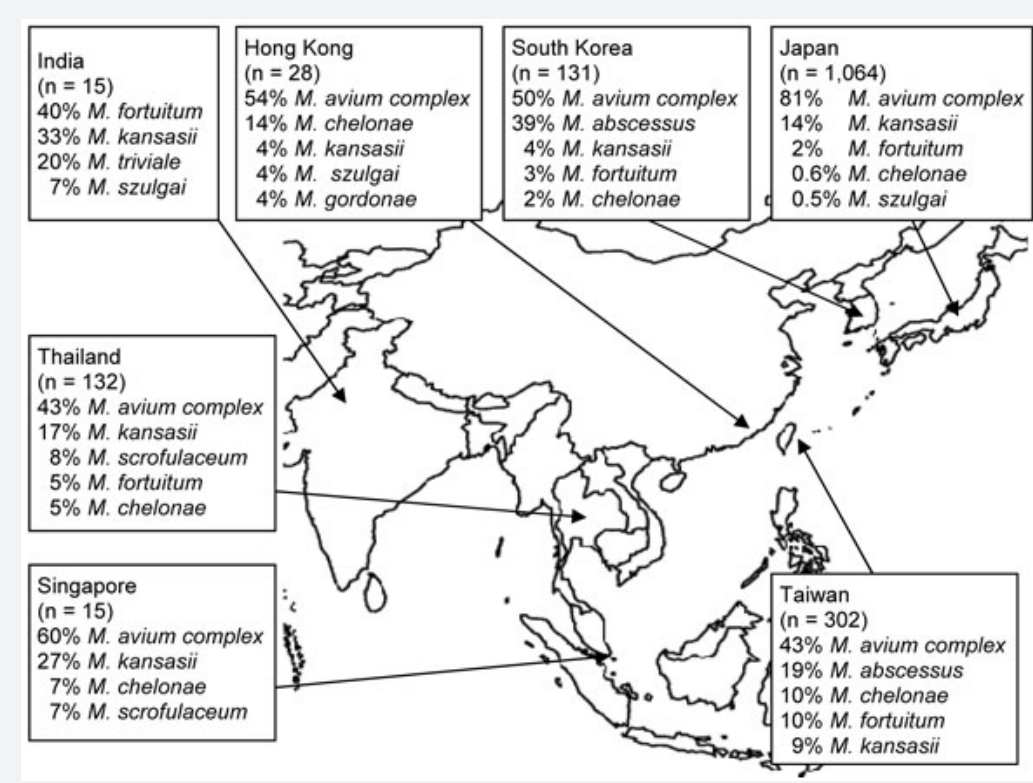

Figure 2: Five most prevalent nontuberculous mycobacteria species found in respiratory specimens, regardless of clinical relevance, Asia, 1971-2007 [17].

In The United States, most of the isolates were M. avium, M. marinum and Granulomatous inflammations are the most common presentation of infection by non tuberculous mycobacteria and are often characterized by localized inflamed lymph nodes [18]. It usually appears as a small superficial nodular lesion but causes severe ulcerative skin disorder with or without hemorrhages in aquatic organisms, animals and human subjects. Since water serves as the natural habitat for many aquatic organisms including different fish species, mycobacteria behaves like an opportunistic pathogen and frequently causes granulomatous skin lesions all over the body with symptoms as progressive weight loss due to loss of appetite, a distended abdomen, fin erosion, unusual coloration, pop-eye and occasionally spinal deformities and chronic infection outbreaks causing mass mortalities [19]. In addition to aquatic infections, fish handlers working in sewage treatment plants, improperly sanitized swimming pools, reservoirs, fish culture systems, industrial processing wastes and even contaminated aquarium or fish tanks are prone to infection on skin, soft tissues and sometimes the exposed bones, the disease being termed as swimming pool Granuloma, Fish Tank Granuloma, Fish Handler's Disease, Fish Handler's Nodules. About twenty species of NTM have been reported to cause granulomatous infection in fish as well as people (fish handlers), where and $M$. chelonae 
are the most virulent. Similar reports have also come in from Indian states $[9,10]$. Infection most commonly occurs at sites of injured skin or external abrasions [20]. All possible signs of this infection includes granulomatous or erythromatous inflammation usually on the fingers or hands represented by a single nodule or non healing open ulcer or a series of multiple nodules with whitish pus. Clinical diagnosis of mycobacterial infection is now confirmed by culture of biopsy material, even if no acid fast bacilli are seen on microscopy. As per Chau Nguyen, NTM infection can serve as an occupational hazard for aquarium cleaners, fishermen, and seafood handlers in poorly sanitized conditions. These drug resistant pathogens from environment might enter nosocomial surroundings through anthropogenic pollution and can cohabit the MTB infections, thus disorienting the drug regime.

The occurrence of nontuberculous mycobacterium are now surprisingly on an increase. The treatment strategies are also evolving simultaneously with different antimycobacterial drug therapy including isoniazid, ethionamide, thiacetazone, rifampin, rifabutin, rifapentine, pyrazinamide, streptomycin, kanamycin, amikacin, capreomycin, gentamicin, tobramycin, ethambutol among others [21]. Previously, the therapeutic treatment for NTM infection in nosocomial environment was done through the administration of various antituberculous drugs, but gradually antibiotic over use has contributed to them becoming resistant to a wide spectrum of antibiotics and disinfectants, raising the concern among medical practitioners and environmentalists. Several cases of mycobacterium infection have demonstrated the development of MDR-M. fortuitum during treatment of pulmonary infection with multiple drugs [22]. This M. fortuitum sample was initially sensitive when treated with a single drug, but subsequently became resistant to multiple drug therapy. Furthermore, amtimycobacterial drugs like pyrazinamide and isoniazid showed effectiveness against various mycobacterial species viz. M. tuberculosis complex but not towards the non tuberculous mycobacteria and even the other members of MTB Complex e.g. M. bovis, whereas ethambutol exerted significant efficacy against MTB complex and various species of NTMs $[23,24]$.

With the increasing health care challenges caused by NTM infections in particular and pathogens in general throughout the developing countries including India, treatments with antibiotics should be restricted, not only due to the growing resistance to multiple drugs, both single and broad spectrum but also due to adverse side effects and higher expense. Overall increase in the Multi-drug resistant and Extreme-drug resistant strains of NTMs and $M$. tuberculosis have complicated the treatment strategies. To overcome these emerging issues, there is an urgent need to study these organisms in detail, document the infections and cases, statistically evaluate the progression of the pathogenic and resistance traits and to develop an alternative therapy which is target oriented, effective, environment friendly and economical, especially keeping in mind the weaker and underprivileged sections of the society.

\section{References}

1. Le Dantec C, Duguet JP, Montiel A, Dumoutier N, Dubrou S, et al. (2002) Occurrence of mycobacteria in water treatment lines and in water distribution systems. Appl Environ Microbiol 68 (11): 5318-5325.

2. Hruska K, Kaevska M (2012) Mycobacteria in water, soil, plants and air: a review. Veterinarni Medicina 57(12): 623-679.

3. Falkinham JO (2009) Surrounded by mycobacteria: nontuberculous mycobacteria in the human environment. J Appl Microbiol 107(2): 356-367.

4. Shukla S, Sharma R, Shukla SK (2013) Detection and identification of globally distributed mycobacterial fish pathogens in some ornamental fish in India. Folia Microbiol (Praha) 58(5): 429-436.

5. Prearo M, Zanoni RG, Campo Dall'Orto B, Pavoletti E, Florio D, et al. (2004) Mycobacterioses: emerging pathologies in aquarium fish. Vet Res Commun 28(1): 315-317.

6. Das P, Saha T, Sengupta T (2016) A detailed report on mycobacteriosis in Pampus argenteus along the Bay of Bengal coast, West Bengal, India. Journal of Coastal Life Medicine 4(10): 930-934.

7. Jain S, Sankar MM, Sharma N, Singh S, et al. (2014) High prevalence of non-tuberculous mycobacterial disease among non-HIV infected individuals in a TB endemic country - experience from a tertiary center in Delhi, India. Pathog Glob Health 108(2): 118-122.

8. Maiga M, Siddiqui S, Diallo S, Diarra B, Traore B, et al. (2012) Failure to recognize nontuberculous Mycobacteria leads to misdiagnosis of chronic pulmonary tuberculosis. PLoS ONE 7(5):e36902.

9. Sengupta T, Das P, Saha T, Abraham JT (2012) A preliminary report on the Mycobacteriosis-like infection among the fish handlers of West Bengal, India. Int J Biol Med Res 3(4): 2648-2650.

10. Saha T, Das P, Sengupta T, Banerjee A (2015) Screening of Aeromonads as associated pathogens from Non Tuberculous Mycobacterial infections in the aquaculture industry, West Bengal, India. Journal of Advanced Laboratory Research in Biology 4(4): 1-6.

11. Braback M, Riesbeck K, Forsgren A (2002) Susceptibilities of Mycobacterium marinum to gatifloxacin, gemifloxacin, levofloxacin, linezolid, moxifloxacin, telithromycin, and quinupristin-dalfopristin (Synercid) compared with its susceptibilities to reference macrolides and quinolones. Antimicrob Agents Chemother 46(4): 1114-1116.

12. Jani MN, Rodrigues C, Mehta AP (2009) The neglected and often ignored: nontuberculous Mycobacteria. J Glob Infect Dis 3(1): 94.

13. Narang R, Narang P, Mendiratta DK (2009) Isolation and identification of nontuberculous mycobacteria from water and soil in central India. Indian J Med Microbiol 27(3): 247-250.

14. Parashar D, Chauhan DS, Sharma VD, Chauhan A, Chauhan SV, et al. (2004) Optimization of procedures for isolation of mycobacteria from soil and water samples obtained in northern India. Appl Environ Microbiol 70(6): 3751-3753.

15. Decostere A, Hermans K, Haesebrouck F (2004) Piscine mycobacteriosis: a literature review covering the agent and the disease it causes in fish and humans. Vet Microbiol 99(3-4): 159-166.

16. Rehulka J, Kaustová J, Řehulková E (2006) Causal agents of mycobacterial diseases in freshwater ornamental fish and their importance for human health in the Czech Republic. Acta Vet Brno 75(2): 251-258. 
17. Simons S, van Ingen J, Hsueh PR, Van Hung N, Dekhuijzen PN, et al. (2011) Nontuberculous Mycobacteria in Respiratory Tract Infections. Emerg Infect Dis 17(3): 343-349.

18. Marras TK, Daley CL (2002) Epidemiology of human pulmonary infection with non-tuberculous mycobacteria. Clin Chest Med 23(3): 553-567.

19. Balouet G, Baudin Laurencin F (1986) Granulomatous nodules in fish: an experimental assessment in rainbow trout, Salmo gairdneri Richardson, and turbot, Scophthalmus maximus (L.). J Fish Dis 9: 417 429.

20. Marzouk MSM, Essa MAA, El-seedy FR, Kenawy AM, El-Gawad DMA (2009) Epizootiological and histopathological studies on mycobacteriosis in some ornamental fishes. Glob Vet 3(2): 137-143.
21. Chau Nguyen (2004) Mycobacterium marinum. The New England Journal of Medicine 350: e8.

22. Dega H, Bentoucha A, Robert J, Jarlier V, Grosset J (2002) Bactericidal activity of rifampicin-amikacin against Mycobacterium ulcerans in mice. Antimicrob Agents Chemother 46(10): 3193-3196.

23. Ruiz-Serrano MJ, Alcala L, Martinez L, Diaz M, Marin M, et al. (2000) In vitro activities of six fluoroquinolones against 250 clinical isolates of Mycobacterium tuberculosis susceptible or resistant to first-line antituberculosis drugs. Antimicrob. Agents Chemother 44(9): 25672568.

24. Wallace RJ, Glassroth J, Griffith DE, Olivier KN, Cook JL, et al. (1997) Diagnostic and treatment of disease caused by nontuberculous mycobacteria. Am J Respir Crit Care Med 156(2 Pt 2): S1-S25.
This work is licensed under Creative Commons Attribution 4.0 Licens

\section{Your next submission with Juniper Publishers}

will reach you the below assets

- Quality Editorial service

- Swift Peer Review

- Reprints availability

- E-prints Service

- Manuscript Podcast for convenient understanding

- Global attainment for your research

- Manuscript accessibility in different formats

( Pdf, E-pub, Full Text, Audio)

- Unceasing customer service

Track the below URL for one-step submission

https://juniperpublishers.com/online-submission.php 